\title{
Fixed field alternating gradient accelerator with small orbit shift and tune excursion
}

\author{
Suzanne L. Sheehy, Ken J. Peach, and Holger Witte \\ John Adams Institute for Accelerator Science, University of Oxford, Oxford, OX1 3RH, United Kingdom \\ David J. Kelliher and Shinji Machida* \\ ASTeC, STFC Rutherford Appleton Laboratory, Harwell Science and Innovation Campus, Didcot, Oxfordshire, OX11 OQX, \\ United Kingdom \\ (Received 20 January 2010; published 22 April 2010)
}

\begin{abstract}
A new design principle of a nonscaling fixed field alternating gradient accelerator is proposed. It is based on optics that produce approximate scaling properties. A large field index $k$ is chosen to squeeze the orbit shift as much as possible by setting the betatron oscillation frequency in the second stability region of Hill's equation. Then, the lattice magnets and their alignment are simplified. To simplify the magnets, we expand the field profile of $r^{k}$ into multipoles and keep only a few lower order terms. A rectangular-shaped magnet is assumed with lines of constant field parallel to the magnet axis. The lattice employs a triplet of rectangular magnets for focusing, which are parallel to one another to simplify alignment. These simplifications along with fringe fields introduce finite chromaticity and the fixed field alternating gradient accelerator is no longer a scaling one. However, the tune excursion of the whole ring can be within half an integer and we avoid the crossing of strong resonances.
\end{abstract}

DOI: 10.1103/PhysRevSTAB.13.040101

PACS numbers: 29.20.- c, 41.85.-p, 29.27.-a

\section{INTRODUCTION}

A fixed field alternating gradient (FFAG) accelerator has the potential for quick acceleration and the delivery of high average intensity beams when the acceleration is repeated sequentially. The acceleration time is determined only by the speed of $\mathrm{rf}$ frequency modulations provided that there is enough rf voltage. Recent demands on high intensity beams and rapid acceleration of unstable particles such as muons and radioactive nuclei have triggered new developments of FFAG accelerators utilizing the availability of modern hardware technology [1].

When invented more than 50 years ago, the optics of the FFAG was designed to keep the transverse tune constant throughout acceleration $[2,3]$. The magnetic field profile was chosen in the form $B_{z}=(r, z=0)=B_{z, 0}\left(r, r_{0}\right)^{k}$, where $r$ and $z$ are radial and vertical coordinates, respectively, and the suffix 0 denotes the reference value. $k$ is called the field index. Either by placing magnets which have an alternating sign of the field strength, or by introducing edge angles with respect to a particle orbit, respectively radial sector-type or spiral sector-type FFAG lattices are realized. Although the local radius of the closed orbit depends on particle momentum, all the orbits become isomorphic. This kind of FFAG accelerator is called a scaling FFAG.

In a scaling FFAG, the field index $k$ determines the focusing strength as well as the amount of orbit shift. The reduction of the orbit shift with larger field index increases the phase advance until the optics become unstable. With a reasonable value for the field index, the orbit

*shinji.machida@stfc.ac.uk shift becomes about $1 \mathrm{~m}$ in typical designs, which makes the lattice magnets larger than that of a conventional synchrotron, although considerably smaller than the magnets of a cyclotron.

Recently, a new kind of FFAG accelerator has been proposed to decrease the orbit shift and also simplify the magnetic field profile [4-6]. The lattice is made from only dipole and quadrupole magnets, which produce, in principle, a linear field instead of the more complicated field profile of the scaling FFAG. The orbit shift is minimized to the order of $\mathrm{cm}$, while keeping the phase advance in a reasonable range. The price we have to pay for this advantage is in tune excursion, i.e., the transverse tune is no longer constant. A beam traverses many integer and halfinteger resonances in the process of acceleration. This type of machine is called a linear nonscaling FFAG.

Beam deterioration due to resonance crossing depends on the strength of the resonance and the crossing speed $[7,8]$. One can easily imagine that the resonance phenomena become more troublesome if the crossing is done over a sufficiently long time scale. In fact, the linear nonscaling FFAG was proposed for muon acceleration where the acceleration is completed within 10 to 20 turns and where the machine tune changes a unit per turn. In this short time scale, we now know that there is no accumulation of orbit distortion due to resonances. Instead, a particle is kicked incoherently when an error field exists in a lattice [9].

Resonance crossing becomes a major problem when the linear nonscaling FFAG is applied to a slower cycling machine such as a proton accelerator for high beam power production or particle therapy accelerators [10,11]. Although the integer and half-integer resonances are all 
nonstructure resonances, the allowed tolerances of the alignment and field profile become very small, such as a few $\mu \mathrm{m}$, and it turns out to be very difficult to construct an accelerator with such tolerances using available technology. Several ways were proposed to introduce chromaticity correction in a linear nonscaling FFAG to keep the transverse tune constant. Johnstone et al. propose using a wedge-shaped quadrupole magnet [12]. The edge focusing and path length of the quadrupole are each made a function of the beam momentum, thereby making the effective focal length of the lattice similar and independent of beam momentum. It was shown that an almost flat tune can be achieved over a wide momentum range-a factor of 6 in momentum is achievable without crossing of major resonances. A more conventional way of introducing chromaticity correction was also studied by adding nonlinear magnets or nonlinear components in the main magnet of a linear nonscaling FFAG [10].

Taking into account the relatively large number of cells and extremely small orbit shift, chromaticity correction in a linear nonscaling FFAG is in fact a challenging problem. First, the upper limit of cell tune variation is inversely proportional to the number of cells. The final goal is to restrict the total tune variation (and not the cell tune variation) to within an integer or less, preferably within half an integer. A small number of cells helps in this respect. Second, a small orbit shift is equivalent to a small dispersion function in a nonlinear magnet. In general, the strength of nonlinearities tends to be strong to correct chromaticity when the dispersion function at the nonlinear magnets is small. It leads to the reduced dynamic aperture. After optimizing the design including chromaticity correction, the resulting machine turns out to be similar to a scaling FFAG in terms of orbit shift and complexity of magnets.

Instead of trying to introduce zero chromaticity in a linear nonscaling FFAG we propose to maintain the properties of a scaling FFAG as much as possible. As well as allowing us to achieve a fixed tune over the momentum range, this approach also allows us to minimize the orbit shifts and simplify the magnets. In fact, we found that the orbit shift of a scaling FFAG could be significantly reduced if the FDF triplet focusing structure was adopted and a large field index in the second stability region of the solution of Hill's equation was used, where $\mathrm{F}$ is a focusing and $\mathrm{D}$ is a defocusing magnet [13]. Although the orbit shift cannot be of the order of $\mathrm{cm}$ like that in a linear nonscaling FFAG, a reduction of about a factor 5 is achieved.

In this paper, we develop this approach further to simplify the magnets and magnet alignment as much as possible despite violating scaling FFAG properties. As shown in the following sections, this leads to an FFAG which combines the small orbit shift and compact magnets of the nonscaling FFAG with the small tune excursion of the scaling FFAG. The small number of cells helps to reduce the total number of magnets and potentially allows the introduction of long straight sections. The design of injection and extraction as well as rf installation would benefit from a long straight. In Sec. II, we will summarize the design of a scaling FFAG with small orbit shift. In Sec. III, several ways are proposed to make the accelerator magnets and alignment simpler by violating the scaling properties and their impacts on optics are discussed. In Sec. IV, we further discuss a method to reduce the tune excursion. We use s-code [14] to fix the geometry of the lattice and ZGOUBI [15] for the optics and tune calculation and particle tracking.

\section{SCALING FFAG WITH SMALL ORBIT SHIFT}

We will summarize here the scaling FFAG design with small orbit shift. In a scaling FFAG, the field index $k$ determines focusing strength as well as orbit shift. A high $k$ value is preferred to decrease the orbit shift, as the orbit shift scales,

$$
\frac{\Delta r}{r_{0}} \cong \frac{1}{k+1} \frac{\Delta p}{p_{0}},
$$

where $\Delta r$ is the orbit shift, $\Delta p$ is the momentum increase, $r_{0}$ and $p_{0}$ are reference orbit radius and momentum, respectively. The upper limit for the $k$ value is determined by limitations in the focusing strength because

$$
\frac{d B_{z}}{d r}=\frac{B_{z}}{r} k
$$

eventually resulting in unstable oscillations.

In fact, there exists another stability region when the focusing strength is increased further in a triplet focusing structure, where the horizontal phase advance per cell is between 180 and 360 degrees and the vertical phase advance is between 0 and 180 degrees. In addition, a simplified lattice model based on Hill's equation suggests the second stability region becomes close to the first stability region when the magnet packing factor is around 0.5 or less. In this case, the lattice beta functions in the second stability region are not significantly different in amplitude from the ones in the first stability region. Even though the second stability region is somewhat more sensitive to machine errors, we find that the allowed errors are still reasonable [13].

Figure 1 shows a stability diagram for stable particle motion for various values of $k$ and the ratio of $\mathrm{F}$ and $\mathrm{D}$ strength. Two main stability regions are identified in the diagram. The first stability region has a horizontal phase advance per cell less than 180 degrees and the second between 180 and 360 degrees. In between the two stability regions a stop band can be observed, which corresponds to 180 degrees phase advance.

We will show the design procedure for the FFAG and some examples based on this principle. As a first step, we fix the magnet packing factor and the cell tunes at 0.4 and 


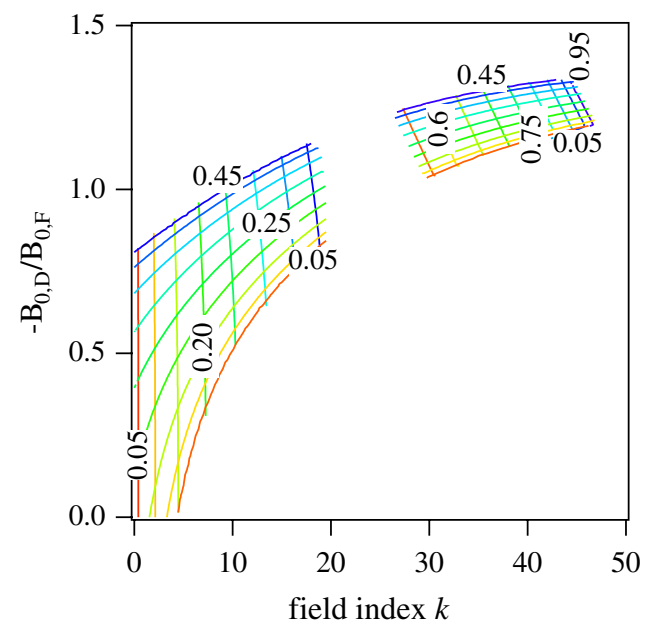

FIG. 1. (Color) From Ref. [13]. Stability diagram shows two stability regions. Upright numbers indicate vertical cell tune and vertically aligned numbers indicate horizontal cell tune. Lines are drawn with 0.05 step.

$(0.75,0.25)$, respectively. Previous studies show that values around those numbers give moderate lattice functions and maximize dynamic aperture. We further assume that the length of each magnet and the space between them is equal. We included the Enge-type fringe field [16]. Under these conditions, the field index $k$ and maximum magnetic field on the beam orbit can be evaluated as a function of cell number as in Table I. The maximum field is normalized for a particle momentum of $1 \mathrm{GeV} / c$ with a reference orbit radius of $10 \mathrm{~m}$.

Second, this table can be scaled for any particle momentum and machine radius. Once the maximum momentum and the maximum allowed field strength are specified, the footprint of a lattice along with other design parameters such as long drift length and orbit shift can be calculated. For example, Table II shows the geometrical lattice parameters for a maximum momentum of $0.729 \mathrm{GeV} / c$, which corresponds to a kinetic energy of $0.250 \mathrm{GeV}$ for a proton, with the maximum field strength of $4 \mathrm{~T}$ on the beam orbit. In order to calculate an orbit shift, we assume that the ratio of the extraction momentum to the injection momentum is 3 .

The choice of cell number depends mostly on hardware restrictions. The number of cells and machine radius should be kept to a minimum to reduce the machine footprint. Drift length should be long enough to accommodate injection, extraction, and rf systems. The orbit shift should be minimized to reduce magnet aperture and cost as a result. Magnet length has less impact, but a smaller ratio of aperture over magnet length is preferable. For this momentum, we have chosen a 12 cell lattice mainly because of a compromise between machine size and orbit shift. This solution is highlighted in bold in Table II. The footprint of the lattice is shown in Fig. 2.

Following the same design principles, the parameters of higher momentum FFAG accelerators are calculated as shown in Tables III, IV, and V. The maximum field strength is $6 \mathrm{~T}$ in the last example. Numbers in boldface indicate the preferred configuration.

The ratio of the extraction momentum to the injection momentum is 3 in all the examples so that it is possible to make a cascade of FFAG accelerators for different momenta, using a lower energy FFAG as an injector. The $0.25 \mathrm{GeV}$ machine can be considered suitable for proton therapy, the $1.5 \mathrm{GeV}$ machine for the accelerator driven subcritical reactor and as a proton driver for neutron pro-

TABLE I. Number of cells and field index $k$ which give the cell tune of $(0.75,0.25)$. Maximum field strength is also shown for a particle momentum of $1 \mathrm{GeV} / c$ with a reference orbit radius of $10 \mathrm{~m}$.

\begin{tabular}{|c|c|c|c|c|c|c|c|c|c|c|c|c|c|c|}
\hline Number of cells & 8 & 12 & 16 & 20 & 24 & 32 & 40 & 48 & 56 & 64 & 80 & 96 & 112 & 128 \\
\hline Field index $k$ & 18.6 & 39.3 & 67.3 & 103 & 145 & 252 & 390 & 560 & 755 & 985 & 1530 & 2200 & 3000 & 3920 \\
\hline Maximum field strength [T] & 2.9 & 3.4 & 3.7 & 3.9 & 4.0 & 4.3 & 4.5 & 4.6 & 4.8 & 4.8 & 5.0 & 5.1 & 5.2 & 5.3 \\
\hline
\end{tabular}

TABLE II. Geometrical lattice parameters as a function of number of cells for a $0.729 \mathrm{GeV} / c$ machine, that is a kinetic energy of $0.250 \mathrm{GeV}$ for a proton.

\begin{tabular}{|c|c|c|c|c|c|}
\hline Number of cells & Radius [m] & Drift length $[\mathrm{m}]$ & Orbit shift [m] & Magnet length [m] & $\mathrm{O} / \mathrm{M}^{\mathrm{a}}$ \\
\hline 8 & 5.376 & 2.533 & 0.293 & 0.338 & 0.868 \\
\hline 12 & 6.251 & 1.964 & 0.168 & 0.262 & 0.642 \\
\hline 16 & 6.751 & 1.591 & 0.108 & 0.212 & 0.508 \\
\hline 20 & 7.126 & 1.343 & 0.075 & 0.179 & 0.418 \\
\hline 24 & 7.376 & 1.159 & 0.055 & 0.154 & 0.358 \\
\hline
\end{tabular}

${ }^{\mathrm{a}} \mathrm{O} / \mathrm{M}$ is the ratio of orbit shift over magnet length. 


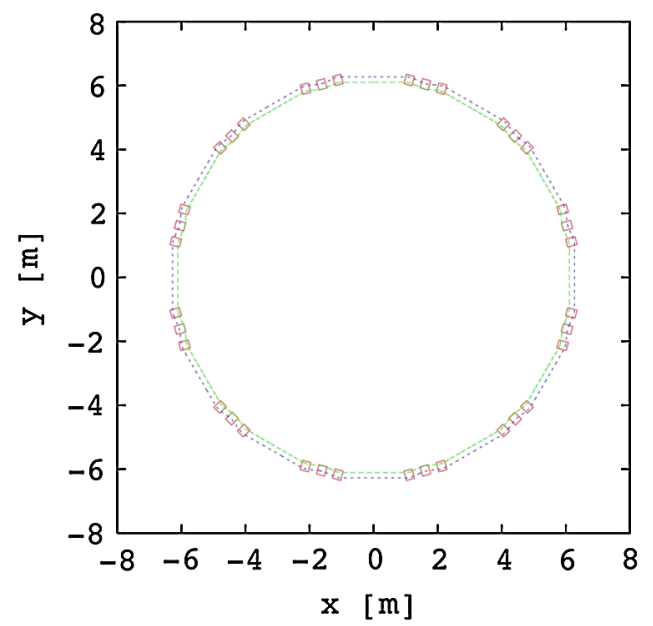

FIG. 2. (Color) Footprint of a 12 cell FFAG. The red rectangles indicate the position of the magnets. Two dotted lines show orbits of $0.243 \mathrm{GeV} / c$ (inside) and 0.729 (outside) $\mathrm{GeV} / c$ proton.

TABLE III. Geometrical lattice parameters as a function of number of cells for a $2.25 \mathrm{GeV} / c$ machine, that is a kinetic energy of $1.5 \mathrm{GeV}$ for a proton.

\begin{tabular}{lccccc}
\hline \hline $\begin{array}{l}\text { Number of } \\
\text { cells }\end{array}$ & $\begin{array}{c}\text { Radius } \\
{[\mathrm{m}]}\end{array}$ & $\begin{array}{c}\text { Drift } \\
\text { length } \\
{[\mathrm{m}]}\end{array}$ & $\begin{array}{c}\text { Orbit } \\
\text { shift } \\
{[\mathrm{m}]}\end{array}$ & $\begin{array}{c}\text { Magnet } \\
\text { length } \\
{[\mathrm{m}]}\end{array}$ & $\mathrm{O} / \mathrm{M}$ \\
\hline 20 & 21.996 & 4.146 & 0.231 & 0.553 & 0.418 \\
$\mathbf{2 4}$ & $\mathbf{2 2 . 7 6 7}$ & $\mathbf{3 . 5 7 6}$ & $\mathbf{0 . 1 7 1}$ & $\mathbf{0 . 4 7 7}$ & $\mathbf{0 . 3 5 8}$ \\
32 & 24.118 & 2.841 & 0.105 & 0.379 & 0.276 \\
\hline \hline
\end{tabular}

TABLE IV. Geometrical lattice parameters as a function of number of cells for a $6.87 \mathrm{GeV} / c$ machine, that is a kinetic energy of $6 \mathrm{GeV}$ for a proton.

\begin{tabular}{lccccc}
\hline \hline $\begin{array}{l}\text { Number of } \\
\text { cells }\end{array}$ & $\begin{array}{c}\text { Radius } \\
{[\mathrm{m}]}\end{array}$ & $\begin{array}{c}\text { Drift } \\
\text { length } \\
{[\mathrm{m}]}\end{array}$ & $\begin{array}{c}\text { Orbit } \\
\text { shift } \\
{[\mathrm{m}]}\end{array}$ & $\begin{array}{c}\text { Magnet } \\
\text { length } \\
{[\mathrm{m}]}\end{array}$ & $\mathrm{O} / \mathrm{M}$ \\
\hline 40 & 76.619 & 7.221 & 0.215 & 0.963 & 0.223 \\
$\mathbf{4 8}$ & $\mathbf{7 9 . 0 9 5}$ & $\mathbf{6 . 2 1 2}$ & $\mathbf{0 . 1 5 5}$ & $\mathbf{0 . 8 2 8}$ & $\mathbf{0 . 1 8 7}$ \\
56 & 81.688 & 5.499 & 0.119 & 0.733 & 0.162 \\
\hline \hline
\end{tabular}

TABLE V. Geometrical lattice parameters as a function of number of cells for a $20.92 \mathrm{GeV} / c$ machine, that is a kinetic energy of $20 \mathrm{GeV}$ for a proton.

\begin{tabular}{lccccc}
\hline \hline $\begin{array}{l}\text { Number of } \\
\text { cells }\end{array}$ & $\begin{array}{c}\text { Radius } \\
{[\mathrm{m}]}\end{array}$ & $\begin{array}{c}\text { Drift } \\
\text { length } \\
{[\mathrm{m}]}\end{array}$ & $\begin{array}{c}\text { Orbit } \\
\text { shift } \\
{[\mathrm{m}]}\end{array}$ & $\begin{array}{c}\text { Magnet } \\
\text { length } \\
{[\mathrm{m}]}\end{array}$ & $\mathrm{O} / \mathrm{M}$ \\
\hline 64 & 168.811 & 9.944 & 0.188 & 1.326 & 0.142 \\
$\mathbf{8 0}$ & $\mathbf{1 7 2 . 6 3 6}$ & $\mathbf{8 . 1 3 5}$ & $\mathbf{0 . 1 2 4}$ & $\mathbf{1 . 0 8 5}$ & $\mathbf{0 . 1 1 4}$ \\
96 & 176.462 & 6.930 & 0.088 & 0.924 & 0.095 \\
\hline \hline
\end{tabular}

duction, and the 6 and $20 \mathrm{GeV}$ ones could be developed as proton drivers for a neutrino factory.

\section{FROM A SCALING TO A NONSCALING FFAG}

In order to make this FFAG accelerator easy to construct, the following simplifications are introduced. First, the radial magnetic field dependence, that is $r^{k}$, is expanded into multipoles expressed as a truncated Taylor series. This is equivalent to multipole fields of the same order, which can be realized in practice for instance by using superconducting magnets. Second, a wedge-shaped magnet, of which both the edges lie along radial lines, is replaced by a rectangular-shaped magnet which makes the structure of the magnet simpler. Third, three rectangular magnets of a triplet focusing cell are aligned parallel with each other on the same girder. In this way, alignment accuracy is considerably increased. These simplifications violate the original requirement of scaling FFAG optics and the accelerator should be called a nonscaling FFAG. The careful adjustment of parameters can, however, minimize the tune excursion as a function of momentum as we will see later on. In the following subsections, those simplifications are incrementally added. Notice that the magnet packing factor in the following design is changed to 0.48 to reduce the maximum field strength.

\section{A. Multipole expansion and truncation}

The magnetic field profile $B_{z}$ can be expressed as

$$
\begin{aligned}
B_{z} & =B_{z 0}\left(\frac{r_{0}+r}{r 0}\right)^{k} \\
& =B_{z 0}\left(1+\sum_{n=1} \frac{1}{n !} \frac{k(k-1) \cdots(k-n+1)}{r_{0}{ }^{n}} r^{n}\right) .
\end{aligned}
$$

The radius of expansion $r_{0}$ is determined as the middle point of the orbit shift at the center of $F$ and $D$ separately when the field profile without truncation is employed.

It is kept the same in the following study. The Enge-type fringe field with a constant extent of $60 \mathrm{~mm}$ is assumed. The magnet is wedge-shaped and aligned such that three triplet magnets face the machine center as that in a scaling FFAG.

In practice, it is desirable to limit the multipole order; depending on the behavior of tune excursions as shown in Fig. 3 where multipoles up to $n=3,4,5$ (octupole, decapole, and dodecapole, respectively) are included. The maximum variation of the total horizontal tune with multipoles up to decapole is 0.056 and of the total vertical tune is 0.029 , both of which are well within half an integer of total tune.

In the case where terms of the multipole expansion up to and including the decapole component are included, the effects of the two extreme field falloff shapes are also examined. In one case the extent of the falloff is proportional to orbit radius whereas in the other case the extent is 

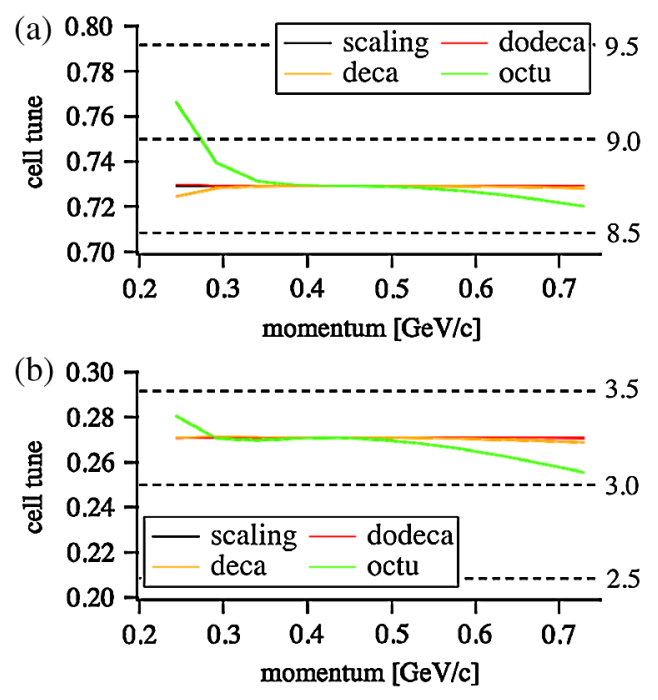

FIG. 3. (Color) Cell tunes throughout acceleration for the case of wedge-shaped magnets with different order of truncation. Horizontal cell tune in (a) and vertical cell tune in (b). Dashed lines and associated numbers show total tune of a 12 cell ring.
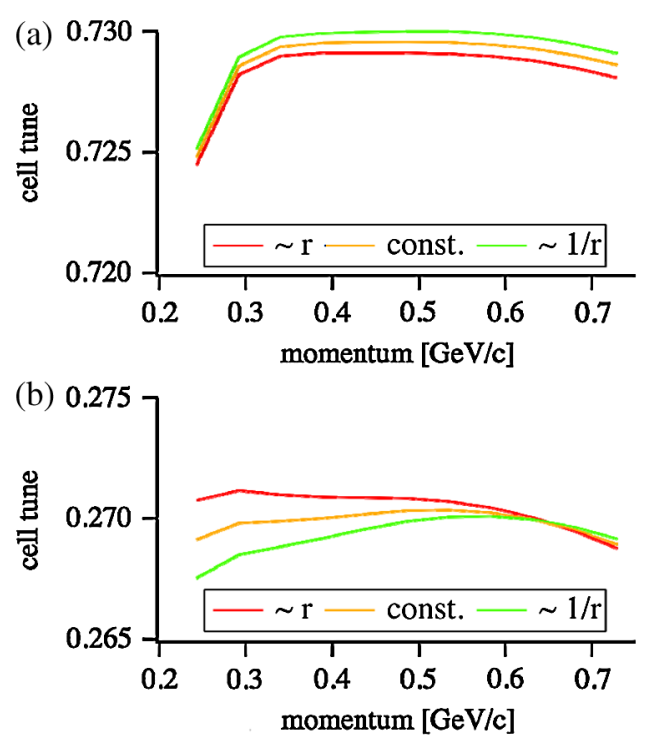

FIG. 4. (Color) Cell tunes throughout acceleration for the case of wedge-shaped magnets with different models of the fringe field falloff. Horizontal cell tune in (a) and vertical cell tune in (b). Notice that the scale of ordinate is 10 times smaller than Fig. 3.

inversely proportional to orbit radius [17]. The difference is small as shown in Fig. 4.

\section{B. Rectangular magnet}

The lattice magnets become simpler to construct and align when they are rectangular rather than wedge or sector shaped. For the purpose of studying the specific effect that using rectangular magnets has on lattice dynamics, the orientation of the magnets remains the same at this stage

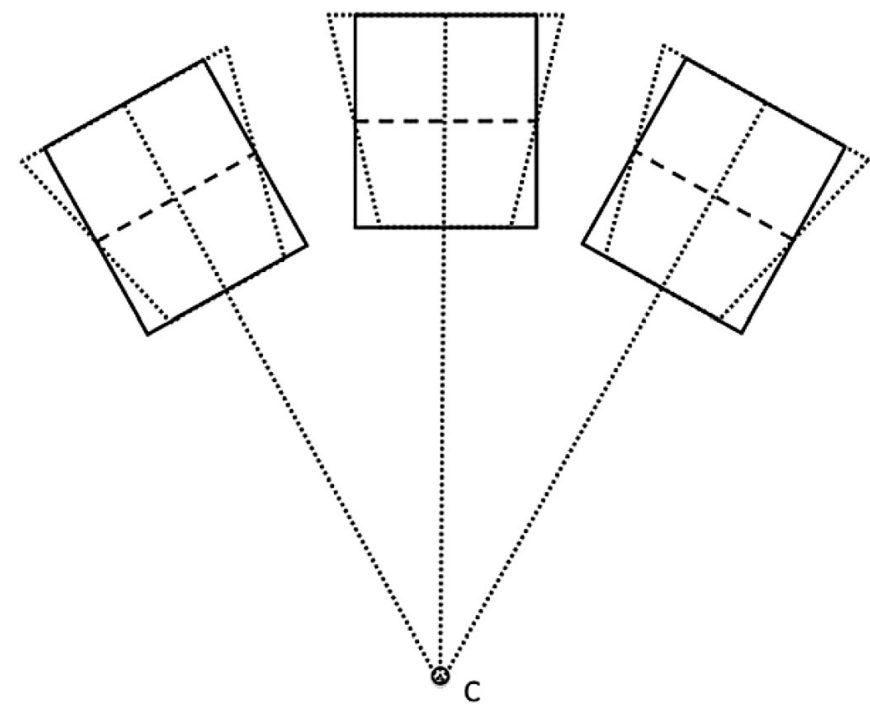

FIG. 5. Converting wedge-shaped magnets (dotted line) to rectangular magnets (solid line). The magnet center is unchanged and the three magnets face the machine center.

so that the three rectangular magnets face the machine center.

An idealized rectangular magnet can be defined with field lines parallel to the magnet axis. The field profile is represented in a Cartesian coordinate system as

$$
\begin{aligned}
B_{z} & =B_{z 0}\left(\frac{y_{0}+y}{y_{0}}\right)^{k} \\
& =B_{z 0}\left(1+\sum_{n=1} \frac{1}{n !} \frac{k(k-1) \cdots(k-n+1)}{y_{0}{ }^{n}} y^{n}\right),
\end{aligned}
$$

where $y_{0}$ at the $\mathrm{F}$ magnet and $\mathrm{D}$ magnet are the same as $r_{0}$ defined in the previous section. Multipoles up to $n=3,4$, 5 (octupole, decapole, and dodecapole, respectively) are included. A line perpendicular to each $\mathrm{F}$ magnet axis intersects that of the D magnet at the machine center, as shown in Fig. 5. The Enge-type fringe field with a constant extent of $60 \mathrm{~mm}$ is assumed. As the magnets are rectangular, it is reasonable to assume that the fringe field extent is constant and independent of radial position.

The resulting betatron tunes throughout acceleration are shown in Fig. 6. The maximum variation of the total horizontal tune with multipoles up to decapole is 0.042 and of the total vertical tune is 0.299 , both of which are well within half an integer of total tune.

\section{Parallel alignment}

To further simplify alignment issues, the three multipole magnets are aligned parallel with each other. As shown in Fig. 7, both $\mathrm{F}$ magnets are rotated with respect to the magnet center so that the three magnets become parallel. This will lead, in a realistic scenario, to a transverse offset 

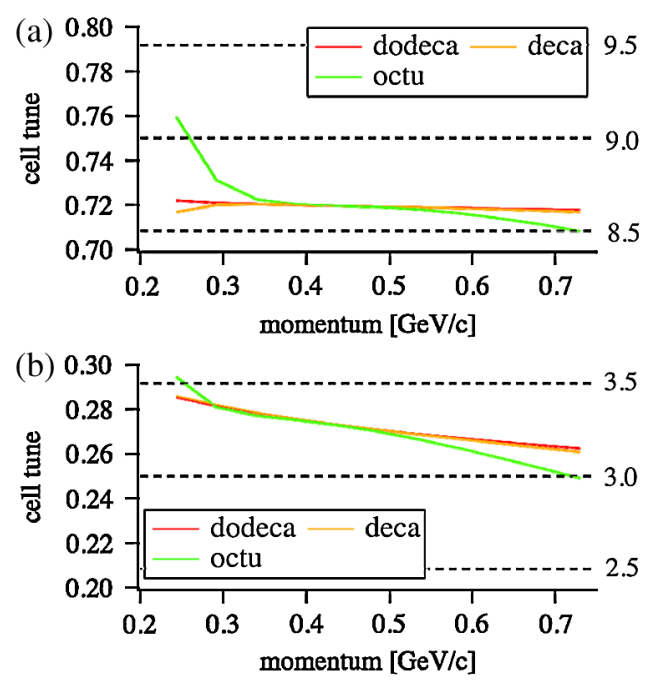

FIG. 6. (Color) Cell tunes throughout acceleration for the case of rectangular-shaped magnets with different order of truncation. Three magnets face the machine center as in Fig. 5. The horizontal cell tune is shown in (a) and the vertical cell tune in (b). Dashed lines and associated numbers show total tune of a 12 cell ring.

between the magnets in the triplet in order to optimize the magnetic bore size. The field profile of each magnet is the same as in the previous section, namely the multipoles up to a certain order are included and the fringe field has a constant extent of $60 \mathrm{~mm}$.

The resulting tunes throughout acceleration are shown in Fig. 8. In the case up to decapole, the maximum variation of the total horizontal tune is 0.092 and of the total vertical tune is 0.250 . These values are very similar to the case before and well within half an integer.
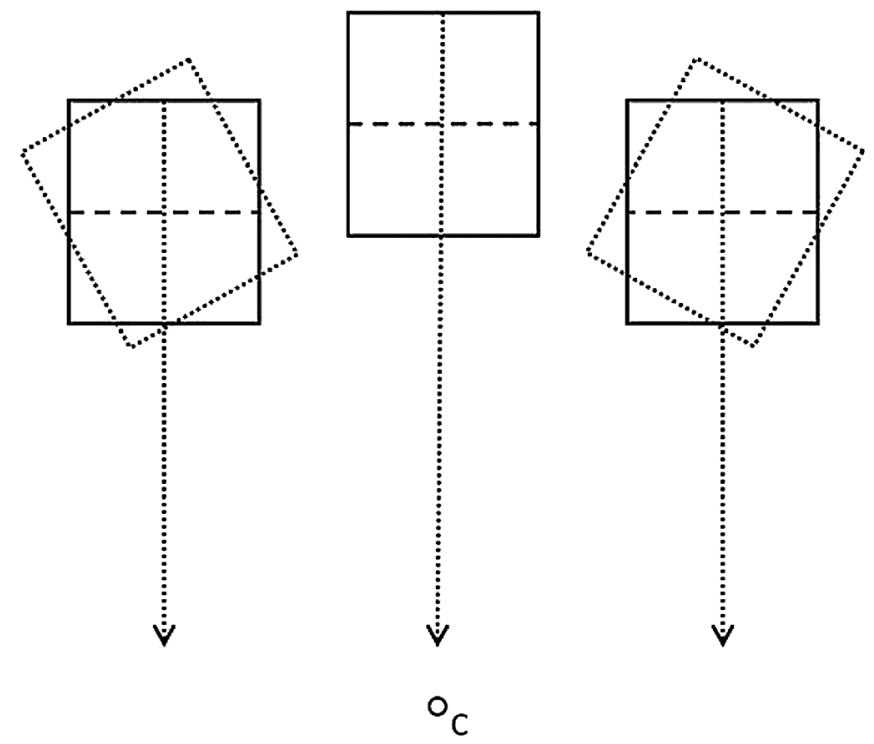

FIG. 7. Converting rectangular magnets facing the machine center (dotted line) to rectangular magnets aligned parallel with each other (solid line).
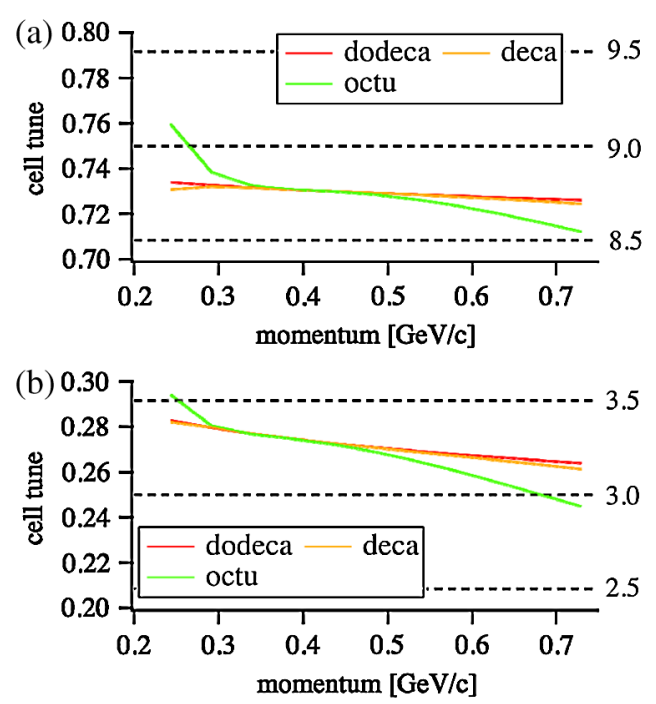

FIG. 8. (Color) Cell tunes throughout acceleration for the case of rectangular-shaped magnets with different order of truncation. Three magnets are aligned parallel with each other. Horizontal cell tune in (a) and vertical cell tune in (b). Dashed lines and associated numbers show total tune of a 12 cell ring.

\section{Dynamic aperture}

A calculation of the dynamic aperture in the case of rectangular magnets with parallel alignment is made. The calculation covers a cell tune range of $0.70-0.75$ and $0.25-$ 0.30 in the horizontal and vertical plane, respectively. In each of these scans, the tune in just one transverse plane is varied while in the other transverse plane it is fixed at the nominal value described above. The calculation is made at injection energy to study the case where the beam is at its largest size in physical space. An error-free lattice is assumed and multipole components up to decapole are included. To select a particular value in the tune space, the field index $k$ (and hence the coefficients of each multipole term) and the ratio of the $\mathrm{F}$ and $\mathrm{D}$ strength are adjusted.

The search for dynamic aperture begins by tracking for 1000 turns a single particle that has identical starting conditions in both planes - in each case the initial coordinate is given by $-\sqrt{2 J / \gamma_{x, y}}$ and the initial angle is zero. $J$ is the action variable and $\gamma_{x, y}$ is the horizontal and vertical Twiss parameter. The tracking is started at $2 J=$ $1 \pi \mathrm{mm}$ mrad normalized amplitude and then increased in steps of $1 \pi \mathrm{mm}$ mrad until the particle is lost. The dynamic aperture is given by the highest amplitude particle that survives tracking.

It is clear from the results shown in Fig. 9 that it is possible to choose a point in the tune space where the dynamic aperture is more than $30 \pi \mathrm{mm} \mathrm{mrad} \mathrm{normalized}$ in both transverse places, which is sufficient for our purposes. One of the local minima in the dynamic aperture results in Fig. 9 can be attributed to a coupling between the transverse planes where the sum of the transverse tunes is 


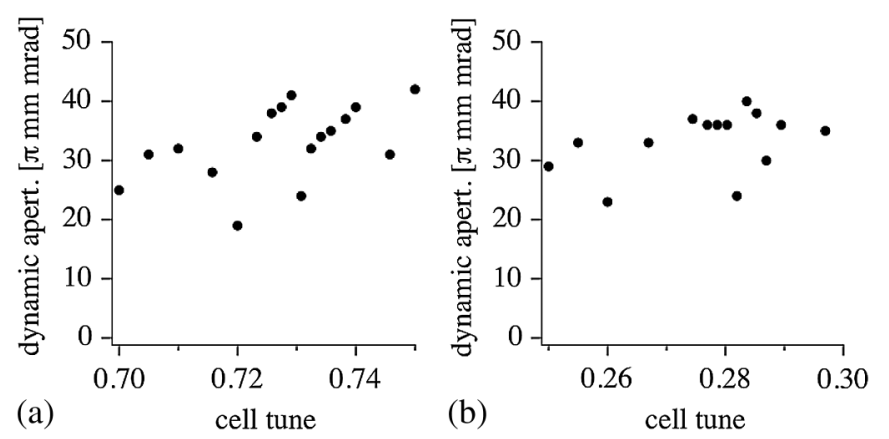

FIG. 9. Dynamic aperture vs (a) horizontal cell tune $q_{x}$, (b) vertical cell tune $q_{y}$. The figure shows a sudden decrease of the dynamic aperture around a horizontal cell tune of 0.720 and around a vertical cell tune of 0.282 , in both cases corresponding to a coupling resonance of $2 q_{x}+2 q_{y}=2$.

close to unity. Since there is no skew quadrupole component present, we can discount a resonance at $q_{x}+q_{y}=1$. Instead it is proposed that the octupole term driving a resonance at $2 q_{x}+2 q_{y}=2$ is responsible for the observed coupling.

\section{DISCUSSION}

So far, the multipole components used have been determined by using a Taylor expansion of the scaling field around the central orbit position for each magnet. However, for an orbit shift of greater than $\sim 100 \mathrm{~mm}$, a closer fit to the field profile of $r^{k}$ can be obtained using a polynomial fit in the region of interest, that is the region of magnetic field experienced by the particle beam. The polynomial fit is calculated using the well-known method of least squares.

The main reason for trying to match the multipole expansion to that of the ideal field profile is to achieve flat tunes throughout acceleration. Since the betatron tunes are determined by the local gradient of the field rather than the field value itself, an improvement may be achieved in the overall tune excursion throughout acceleration, by ensuring that the gradient of the field is as close as possible to the gradient of the ideal profile. The variation from the ideal gradient profile after using a Taylor expansion to decapole is shown in Fig. 10. The gradient of the field differs from the exact scaling profile by around $1 \%$ at the low momentum end, where the maximum tune excursion is observed in Fig. 3.

Therefore, in order to achieve flatter tunes throughout acceleration, we consider using a polynomial to fit to either the field strength or field gradient determined by the scaling law. The resulting tune excursion for polynomial fits to the field strength and field gradient using up to the decapole component, and the tune excursion for a polynomial fit to the field gradient using only up to the octupole component, are shown in Fig. 11.

These results indicate that by simply using a polynomial fit to either the field profile or the gradient instead of using a Taylor expansion results in a greatly improved fit to the local field gradient, and hence a much reduced total tune excursion. Dynamic aperture of this lattice is similar to that

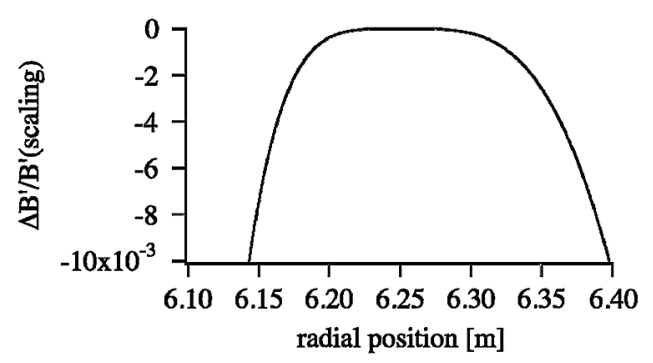

FIG. 10. Difference in field gradient between Taylor expanded scaling field and exact scaling field.
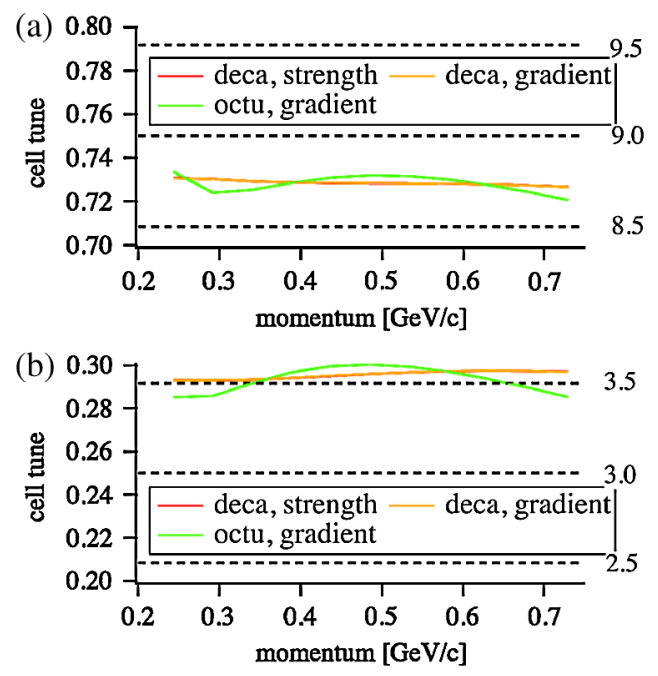

FIG. 11. (Color) Cell tunes throughout acceleration for the case of rectangular-shaped magnets with polynomial fit to either strength or gradient of scaling field. Three magnets are aligned parallel with each other. Horizontal cell tune in (a) and vertical cell tune in (b). Dashed lines and associated numbers show total tune of a 12 cell ring. In both (a) and (b) the difference between the two decapole cases is too small to be visible at this scale.
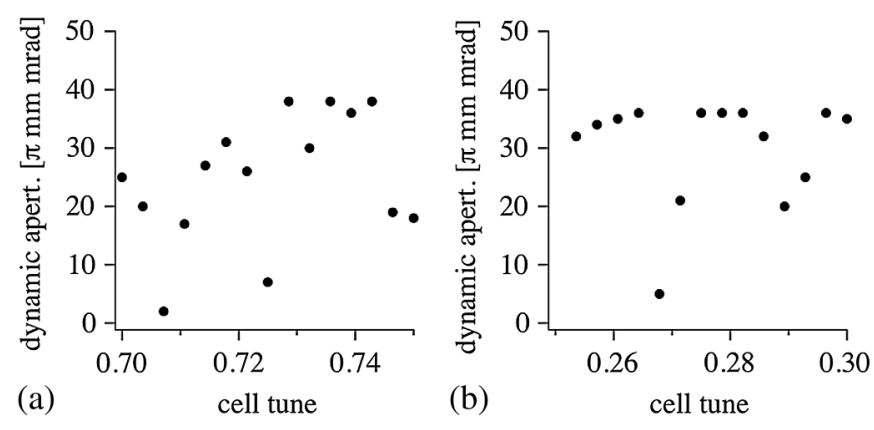

FIG. 12. Dynamic aperture vs (a) horizontal cell tune $q_{x}$, (b) vertical cell tune $q_{y}$. The lattice of rectangular-shaped magnets is used with polynomial fit to the strength up to decapole. 
of the lattice with the simple multipole expansion as shown in Fig. 12.

In fact, the variation of the total tune throughout acceleration using a polynomial fit up to the octupole component is reduced to just 0.156 and 0.182 for the horizontal and vertical tunes, respectively. These are both well within half an integer, indicating that it is possible to achieve the required total tune constraint without the need for a decapole field if a polynomial fit to the field profile is used. This allows for further simplification of the required magnets, helping to reduce the machine cost.

\section{SUMMARY}

A nonscaling FFAG with small orbit shift and tune excursion was designed based on a scaling FFAG with a high field index $k$. It uses rectangular magnets with a few multipole components. The lattice magnets are aligned parallel with each other, which makes alignment simple. The multipole components are optimized so that the tune excursion during acceleration fits well within half an integer of the total tune. This FFAG accelerator is free from major resonances. Dynamic aperture is large enough for most applications as a proton accelerator.

\section{ACKNOWLEDGMENTS}

The work is supported by the U.K. Neutrino Factory/ Particle Physics and Astronomy Research Council (PPARC) under Contract No. 2054 and the CONFORM project/Engineering and Physical Sciences Research Council (EPSRC) under Grant No. EP/E032869/1. We would like to thank Dr. C. R. Prior, Dr. G. H. Rees, and Dr. T. Yokoi for their fruitful discussions and continuous encouragement. We appreciate Dr. F. Méot's help to use his code ZGOUBI.

\section{APPENDIX: ZGOUBI DESCRIPTION OF A RECTANGULAR MAGNET}

We used the code ZGOUBI as well as s-code to calculate optics for different configurations. Unfortunately, ZGOUBI has only a model of a wedge-shaped magnet in a polar coordinate system for a FFAG-type field profile. We will describe a way to model a rectangular-shaped magnet with the existing ZGOUBI commands. In the following, we use the same symbols defined in the ZGOUBI [18].

Wedge- or sector-shaped magnets for a FFAG-type field profile can be modeled in ZGOUBI using the magnet element DIPOLES which uses a polar coordinate system and has a magnetic field of the following form:

$$
\begin{aligned}
B_{z}(R, \theta)= & B_{z 0} F(R, \theta)\left[1+b_{1}\left(\frac{R-R M}{R M}\right)\right. \\
& \left.+b_{2}\left(\frac{R-R M}{R M}\right)^{2}+b_{3}\left(\frac{R-R M}{R M}\right)^{3}+\cdots\right],
\end{aligned}
$$

where $B_{z 0}$ is a field at radius $R M, F(R, \theta)$ is the fringe field coefficient, and $b_{i}$ is the $i$ th multipole coefficient. The fringe field coefficient is calculated as

$$
F(R, \theta)=\frac{1}{1+\exp P(d)},
$$

where $d$ is the distance to the magnet edge and

$$
\begin{aligned}
P(d)= & C_{0}+C_{1}\left(\frac{d}{g}\right)+C_{2}\left(\frac{d}{g}\right)^{2}+C_{3}\left(\frac{d}{g}\right)^{3}+C_{4}\left(\frac{d}{g}\right)^{4} \\
& +C_{5}\left(\frac{d}{g}\right)^{5} .
\end{aligned}
$$

In addition to that, the dependence of the $g$ on $R$ is given by the $\kappa$ parameter defined as follows:

$$
g(R)=g_{0}\left(\frac{R M}{R}\right)^{\kappa} .
$$

As typical values for a conventional magnet, we use $C_{0}=$ $0.1455, C_{1}=2.267, C_{2}=-0.6395$, and $C_{4}=C_{5}=0$. We set $\kappa=0$ because of the argument we discussed before.

The magnet element DIPOLES is positioned using radial coordinate $R=R E$ and angle $T E$ to define the entrance to the magnet and $R S, T S$ to define the exit. When a trajectory is perpendicular to the magnet face, we define $T E, T S=0$. At the exit, a reference particle goes through a position at $R=R S$ with an angle of $T S$. Between the entrance and the exit, longitudinal magnet length is specified by the angular extent $A T$ in a polar coordinate system. Usually, $R M, R E$, and $R S$ should have similar values because a beam is supposed to go around near the center of multipole expansion at $R=R M$.

A rectangular magnet is modeled using DIPOLES with very large $R E$ and $R S$ and very small $A T$. By using the same scaling factor, longitudinal magnet length is kept constant. For example, we found that optics properties converge when $R E$ and $R S$ are more than 5000 times larger and $A T$ is smaller by the same amount. $R E$ and $R S$ are no longer the machine radius. In order to have the same multipole strength, the multipole coefficient is multiplied by (the scaling factor) ${ }^{i}$.

To align the three magnets facing the machine center, CHANGEREF command with appropriate angle, which is calculated in the case of wedge-shaped magnets facing the machine center, is introduced between the magnet definitions to rotate the reference coordinate system. To align the three magnets parallel with each other, a CHANGEREF command is introduced only once at the end of the last magnet. In the former case, $R M$ for $\mathrm{F}$ magnet and $R M$ for $\mathrm{D}$ magnet are taken similar to $R E$ with the same relative shift as in the case of wedge-shaped magnets facing the machine center. In the latter, $R M$ for $\mathrm{F}$ magnet and $R M$ for $\mathrm{D}$ magnet are again taken similar to $R E$ with the relative shift equal to the distance between the magnet center of D and the straight line connecting the magnet 
center of both F magnets. The ZGOUBI input files we use can be found in [19].

[1] M. Aiba, K. Koba, S. Machida, Y. Mori, R. Muramatsu, C. Ohmori, I. Sakai, Y. Sato, A. Takagi, R. Ueno, T. Yokoi, M. Yoshimoto, and Y. Yuasa, in Proceedings of the European Particle Accelerator Conference, Vienna, 2000 (EPS, Geneva, 2000), p. 581, http://www.jacow.org/.

[2] K. R. Symon, D. W. Kerst, L. W. Jones, L. J. Laslett, and K. M. Terwillinger, Phys. Rev. 103, 1837 (1956).

[3] A. A. Kolomensky and A. N. Levedev, Theory of Cyclic Accelerators (North-Holland, Amsterdam, 1966), p. 337.

[4] C. Johnstone, W. Wan, and A. Garren, in Proceedings of the 18th Particle Accelerator Conference, New York, 1999 (IEEE, New York, 1999), p. 3068, http://www.jacow.org/.

[5] E. Keil and A. M. Sessler, Nucl. Instrum. Methods Phys. Res., Sect. A 538, 159 (2005).

[6] D. Trbojevic, E. D. Courant, and M. Blaskiewicz, Phys. Rev. ST Accel. Beams 8, 050101 (2005).

[7] R. Baartman, http://www.triumf.ca/ffag2004/.

[8] G. Guignard, CERN Report No. CERN 77-10, 1977.

[9] S. Machida and D. J. Kelliher, Phys. Rev. ST Accel. Beams 10, 114001 (2007).
[10] S. Machida, Phys. Rev. ST Accel. Beams 11, 094003 (2008).

[11] T. Yokoi, J. Cobb, K. Peach, and S. Sheehy, in Proceedings of the 11th European Particle Accelerator Conference, Genoa, 2008 (EPS-AG, Genoa, Italy, 2008), p. 3398, http://www.jacow.org/.

[12] C. Johnstone and S. Koscielniak, in Proceedings of the 10th European Particle Accelerator Conference, Edinburgh, Scotland, 2006 (EPS-AG, Edinburgh, Scotland, 2006), p. 2290, http://www.jacow.org/.

[13] S. Machida, Phys. Rev. Lett. 103, 164801 (2009).

[14] S. Machida, ICFA Beam Dynamics Newsletter No. 43, edited by C. R. Prior (2007), p. 54, http://icfa-usa.jlab. org/archive/newsletter.shtml/.

[15] F. Méot, Nucl. Instrum. Methods Phys. Res., Sect. A 427, 353 (1999).

[16] H. A. Enge, Focusing of Charged Particles, edited by A. Spetier (Academic Press, New York, 1967), Vol. 2, p. 203.

[17] J. Fourrier, F. Martinache, F. Méot, and J. Pasternak, Nucl. Instrum. Methods Phys. Res., Sect. A 589, 133 (2008).

[18] F. Méot and S. Valero, ZGOUBI Users' Guide (2008), http:// sourceforge.net/projects/zgoubi/.

[19] http://www.astec.ac.uk/intbeams/users/machida/ publications/20100120/. 